\title{
Effects of drug-polymer dispersions on solubility and in vitro diffusion of artemisinin across a polydimethylsiloxane membrane
}

\author{
SHAHZAD Yasser ${ }^{1,2^{*}}$, SHAH Syed Nisar Hussain ${ }^{1}$, ANSARI Muhammad Tayyab ${ }^{1}$, \\ RIAZ Romana $^{1}$, SAFDAR Aasma ${ }^{1}$, HUSSAIN Talib ${ }^{1,2} \&$ MALIK Madeeha ${ }^{3}$ \\ ${ }^{1}$ Faculty of Pharmacy, Bahauddin Zakariya University, Multan 60800, Pakistan; \\ ${ }^{2}$ Division of Pharmacy and Pharmaceutical Sciences, School of Applied Sciences, University of Huddersfield, Queensgate, Huddersfield HD1 3DH, \\ $U K$; \\ ${ }^{3}$ Hamdard Institute of Pharmaceutical Sciences, Hamdard University, Islamabad 44220, Pakistan
}

Received August 30, 2011; accepted October 18, 2011

\begin{abstract}
Artemisinin (ART) is a sesquiterpene lactone with an endo-peroxide bridge that is thought to be responsible for its antimalarial activity. It has low oral bioavailability because of aqueous insolubility, which leads to local toxicity at the site of aggregation. The present work focused on increasing its solubility and evaluating its permeation across a model membrane to mimic transdermal delivery that bypasses the hepatic metabolism. For this purpose, physical mixtures (PM), solid dispersions (SD) and lyophilized dispersions (LD) with different drug-polymer ratios $(1: 0.5,1: 1,1: 2,1: 4$ and 1:9) were prepared using the hydrophilic polymer polyvinylpyrrolidone (PVP). Drug-polymer dispersions were characterized using X-ray diffraction (XRD) and Fourier transform infrared spectroscopy (FTIR). Solubility was measured in three solvents: de-ionized water, phosphate buffered saline (PBS) and methanol. The toluene-water partition coefficient was evaluated and compared with the literature and calculated $\log P$ values. In vitro diffusion of ART was studied across a polydimethylsiloxane membrane from a saturated solution of drug-polymer dispersions. XRD patterns showed a gradual decrease in crystallinity of ART with increasing polymer concentration, while FTIR confirmed no interactions between ART and PVP. Solubility was increased up to 4-, 5- and 8-fold for LD in water, PBS and methanol, respectively. The $\log P$ for toluene-water was $2.65 \pm 0.3$, which is in good agreement with literature and calculated $\log P$ values. Permeation was enhanced, which is attributed to the decrease in crystallinity and increase in wettability of the drug. The ART flux was significantly higher than that of pure ART $(0.12 \pm 0.01)$ with increasing PVP concentration for SD and LD formulations. In conclusion, drug-polymer dispersions with PVP improve the pharmaceutical properties of ART in the order LD>SD>PM.
\end{abstract}

artemisinin, solid dispersions, flux, permeability coefficient, polyvinylpyrrolidone, solubility, silicone membrane

Citation: Shahzad Y, Shah S N H, Ansari M T, et al. Effects of drug-polymer dispersions on solubility and in vitro diffusion of artemisinin across a polydimethylsiloxane membrane. Chin Sci Bull, 2012, 57: 1685-1692, doi: 10.1007/s11434-012-5094-2

Resistance to conventional antimalarial drugs has led to changes in malaria control policies globally in favor of artemisinin (ART). ART is a parent compound of a novel family of antimalarials extracted from the Chinese traditional plant, Artemisia annua L. Asteraceae. It has been reported that ART is very effective against malaria parasites, including the multidrug-resistant falciparum species [1,2]. It was first isolated and characterized as an active compound

*Corresponding author (email: y.shahzad@hud.ac.uk) by Chinese scientists and since then has been successfully utilized as an antimalarial drug [3]. ART is a sesquiterpene lactone with an inner peroxide bridge that is responsible for its antimalarial activity, as shown in Figure 1 (taken from CDS, Daresbury UK through freely available ACD software). ART is a poorly water-soluble drug with an octanol-water partition coefficient $(\log P)$ greater than 2 and a short half-life of $2-3 \mathrm{~h}$ and it is extensively metabolized by the liver. Thus, oral bioavailability is low $(32 \%)[1,4,5]$. Although ART has shown excellent permeability across the 


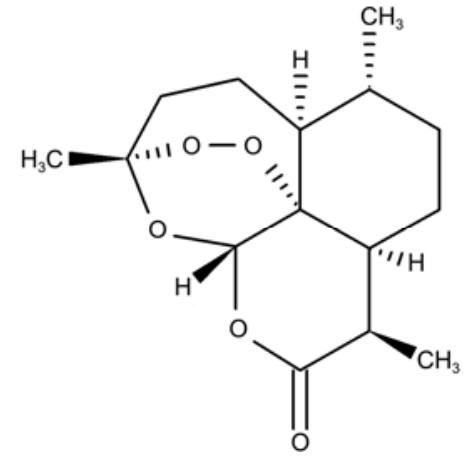

Figure 1 Structure of artemisinin (Chemical Database Service, Daresbury, UK).

intestinal mucosa, it has low bioavailability because of poor aqueous solubility, which may lead to incomplete clearance of the malaria parasite, resulting in recurrence of malarial symptoms [6].

Transdermal drug delivery facilitates the passage of therapeutic quantities of drug substances through the skin into the general circulation where they can have systemic effects, thus bypassing the hepatic first-pass effect. Drug delivery through the cutaneous route has several advantages over other routes but faces a major problem presented by the barrier function of the skin, in which the stratum corneum plays a vital role [7]. Physical and chemical methods are widely used to overcome this passive barrier. The physical enhancement techniques currently in use, for example iontophoresis and sonophoresis, require complex equipment [8]. Alternatively, chemical permeation enhancers are extensively used, which temporarily lower the impermeability of skin and facilitate the absorption of drug through the skin. The physical and chemical properties should be kept in mind whilst selecting a chemical permeation enhancer [9]. By far the most common penetration enhancers in use are ethanol, alcohols with long carbon chains, cyclic monoterpenes, surfactants, pyrrolidones, propylene glycol, isopropyl myristate and dimethyl sulfoxide [10-12].

Various techniques have been used previously to improve the solubility and in turn the dissolution of drugs that are poorly soluble in water. Drug-polymer complexation using a hydrophilic polymer is quite a popular technique and involves formation of solid dispersions using either solvent evaporation or a freeze drying method [13-15]. Attempts had been made to enhance the dissolution rate of ART by making solid dispersions with PVP K-25 and cyclodextrins, resulting in improved dissolution to some extent $[5,16]$. Polyvinylpyrrolidone (PVP) is a water-soluble polymeric compound composed of repeating units of $N$-vinyl pyrrolidone. PVP is well tolerated physiologically and has been used for increasing the solubility and oral absorption of many water insoluble drugs $[17,18]$. Enhancement of the solubility, dissolution and permeation profile of a water insoluble drug using PVP K-30 has been documented [19]. To date, there have been no published reports on the solubility in different solvents and permeation of ART. In the present work, we report the effect of PVP on solubility and in vitro diffusion of ART across a synthetic membrane. The drug-polymer dispersions were also characterized for their solid state properties using Fourier transform infrared spectroscopy (FTIR) and X-ray diffraction (XRD).

\section{Materials and methods}

\subsection{Chemicals}

The following chemicals were used as purchased: artemisinin (ART) 99.9\% purity (Alchem, New Dehli, India); polyvinylpyrrolidone (PVP-k30, Beijing Chemical Reagent Company, China); methanol HPLC grade 99\%+ (Merck, Darmstadt, Germany); potassium dihydrogen phosphate (VWR, Lutterworth, Leicestershire, UK); sodium chloride and potassium chloride (Sigma-Aldrich, Poole, Dorset, UK); disodium hydrogen phosphate (Fischer Scientific Chemicals, Loughborough, Leicestershire, UK); vacuum grease (Dow Corning, Midland, MI, USA).

\subsection{Artemisinin assay}

The concentration of ART was measured by a slight modification of the method described by Zhao and Zeng [20], in which the ART is transformed into a UV-absorbing compound through an alkali reaction, i.e. by heating the solution with $0.2 \% \mathrm{NaOH}$ solution. Appropriate dilutions were prepared from a standard stock solution of ART and the alkali reaction was carried out by adding $5 \mathrm{~mL}$ of $0.2 \% \mathrm{NaOH}$ solution to each dilution, then heated at $50 \pm 1^{\circ} \mathrm{C}$ for $30 \mathrm{~min}$ and allowed to cool down in a refrigerator. The absorbance was measured at $290 \mathrm{~nm}$ with a UV spectrophotometer (Agilent 2005, Germany) and a calibration curve was obtained. The UV assay method was validated for inter- and intra-day variations.

\subsection{Preparation of drug-polymer dispersions}

Table 1 displays all formulation types, along with the corresponding sample identification codes used in this study. Physical mixtures (PMs), solid dispersions (SDs) and lyophilized dispersions (LDs) were prepared by different ART to PVP ratios $(1: 0.5,1: 1,1: 2,1: 4$ and 1:9). Physical mixtures were prepared by thoroughly mixing the drug and polymer in a pre-cleaned and dried glass mortar \& pestle for about 5-10 min until a homogeneous mixture was obtained. Solid dispersions were prepared by the solvent evaporation method, in which an appropriate quantity of PVP was added to a solution of ART $(1 \mathrm{~g})$ in methanol $(100 \mathrm{~mL})$ by continuous stirring in a pre-cleaned vessel. Methanol was evaporated on a rotary evaporator and the dried mass obtained was finally pulverized in a pre-cleaned pestle and mortar for 
Table 1 Formulations with their codes

\begin{tabular}{llllll}
\hline \multirow{2}{*}{ Formulation type } & \multicolumn{5}{c}{$\begin{array}{c}\text { Drug to polymer ratio and corresponding } \\
\text { formulation code }\end{array}$} \\
\cline { 2 - 6 } & $1: 0.5$ & $1: 1$ & $1: 2$ & $1: 4$ & $1: 9$ \\
\hline Physical mixture (PM) & F1 & F2 & F3 & F4 & F5 \\
Solid dispersions (SD) & F6 & F7 & F8 & F9 & F10 \\
Lyophilized dispersions (LD) & F11 & F12 & F13 & F14 & F15 \\
\hline
\end{tabular}

about 5 min until a homogeneous mixture was obtained and then refrigerated in a closed vial at $5^{\circ} \mathrm{C}$ until further investigation.

For LDs, SDs were dissolved in methanol until a clear solution was obtained. This solution was quickly solidified by decreasing the temperature to $-50^{\circ} \mathrm{C}$ by immersing the flask in a cold methanol bath. Upon cooling, the flask was attached to the vacuum adapter of the lyophilizer for sublimation. After the solvent was completely removed, a porous powder residue appeared and was kept in a refrigerator at $5^{\circ} \mathrm{C}$ until further investigation.

\subsection{Characterization of dispersions}

(1) Fourier-transform infrared spectrophotometry. FTIR spectra were measured on a Schimadzu, 2400s Spectrometer using the $\mathrm{KBr}$ disc method. The samples were scanned over the range 400-4000 $\mathrm{cm}^{-1}$.

(2) X-ray powder diffraction. XRD for each ratio of PM, SD and LD was performed as described earlier using a Bruker D8 Discover (Germany) apparatus [21]. Measurement conditions included target $(\mathrm{Cu} \mathrm{K} \alpha)$, voltage $(35 \mathrm{kV})$, and current (35 mA). A system of diverging, receiving, and anti-scattering slits of $1^{\circ}, 1^{\circ}, 0.15^{\circ}$, respectively, was used. Eva software (Evaluation Package Bruker, Germany) was used for data processing. Patterns were obtained using a scan speed of $4^{\circ} / \mathrm{min}$ with $2 \theta$ between $5^{\circ}$ and $50^{\circ}$.

\subsection{Solubility studies and toluene-water partition coefficient}

The solubility of pure ART and PMs, SDs and LDs was determined in three solvents: distilled water, phosphate buffered saline (PBS), and methanol. An excess quantity of each sample was stirred with each of the solvents for $48 \mathrm{~h}$ at a constant temperature of $37 \pm 2{ }^{\circ} \mathrm{C}$. The suspensions were then centrifuged at $4000 \mathrm{r} / \mathrm{min}$ for $30 \mathrm{~min}$ and a supernatant aliquot was taken out by a micro-pipette and analyzed spectrophotometrically at $290 \mathrm{~nm}$ after alkali reaction to determine the concentration. The solubility was also described in terms of the solubility enhancement ratio $\left(\mathrm{ER}_{\mathrm{sol}}\right)$, which is the ratio between drug solubility with the polymer and a standard (pure ART in this case).

Toluene-water partition coefficient was determined using the shake flask method by dissolving $0.1 \mathrm{mg}$ of ART in $10 \mathrm{~mL}$ of a 50:50 toluene water mixture. The flask was agitated for $24 \mathrm{~h}$ and then allowed to stand for $24 \mathrm{~h}$ to completely separate the layers. The amount of drug in each layer was analyzed and the toluene-water $\log P$ was calculated.

\subsection{In vitro diffusional studies across a model membrane}

Diffusion studies of the selected permeant across silicone membrane were performed using Franz-type diffusion cells which were having a receptor phase volume of $\sim 5 \mathrm{~mL}$ and a diffusional area of $\sim 0.788 \mathrm{~cm}^{2}$. [22]. Sheets of silicone membrane were cut to appropriate circular sizes and soaked overnight in the receptor solution. The membrane was mounted between the two compartments of the diffusion cells and vacuum grease was used to produce a leak-proof seal. The receptor compartment was filled with degassed PBS at pH 7.4 (which is near to skin $\mathrm{pH}$ ). To prevent evaporation from the donor and receptor compartment, the donor cell and arm was covered with parafilm. Uniform mixing of the receptor phase was obtained with a magnetic stirrer placed in the receptor compartment. The diffusion cells were placed on a stirring bed (Variomag, USA) immersed in a water bath at $37 \pm 5^{\circ} \mathrm{C}$ to maintain a temperature of $\sim 32^{\circ} \mathrm{C}$ at the membrane surface. After $1 \mathrm{~h}$ the receptor phase was completely removed and refilled with pre-thermostated and degassed PBS. The donor cell was charged with $1 \mathrm{~mL}$ of saturated solution of each ratio, with excess solute present to maintain saturation throughout the experiment. This ensures that the depletion of solute in the vehicle did not become the rate-limiting step. After predetermined time intervals of $5,15,30,45,60,90,120$, and $180 \mathrm{~min}, 0.2 \mathrm{~mL}$ of sample was withdrawn using micro pipette, followed by the addition of same volume of pre-thermostated receptor solution to maintain the sink conditions. The samples were analyzed spectrophotometrically at $290 \mathrm{~nm}$ after alkali reaction to obtain the amount permeated through the silicon membrane. Experiments were conducted in triplicate to assess statistically significant data.

\subsection{Statistical analysis}

All the results presented are expressed as mean $\pm \mathrm{SD}(n=3)$ except for the solubility. One-way ANOVA with $P \leqslant 0.05$ was performed to check the significance in the values obtained and post hoc comparison was carried out using the Turkey multiple test, where the difference in means was significant.

\section{Discussion and conclusions}

The $\log P$ was measured and compared with literature and calculated $(\operatorname{cog} P$ ) values (calculated using ACD labs software, CDS Daresbury, UK). ART is a lipophilic molecule with a $\log P$ value of $2.65 \pm 0.3$. The toluene-water partition 
coefficient obtained in this experiment was in good agreement with the literature $\log P$ (octanol-water) value (2.90) [23] and $\operatorname{cog} P$ value (2.40). Tables $2-4$ describe the solubility of ART in the presence of different concentrations of PVP in the three solvent systems examined in this work. The enhanced effect on the solubility of ART by PVP is also given in terms of the solubility enhancement ratio $\left(\mathrm{ER}_{\mathrm{sol}}\right)$. From the data obtained, it is clear that there is significant enhancement of the solubility of ART by PVP. The improvement in solubility can be explained by two different factors, namely, the PVP solubilizing effect and the type of formulation.

PVP has a tendency to decrease the interfacial tension between solute and solvent, hence increasing the wettability of the solute, which in turn increases the solubility of the test compound [24]. It is evident from the results that enhancement of solubility is dependent on the concentration of polymer. Higher polymer concentrations result in higher solubilities of ART. A similar effect was observed in a study by El-Badry and Fathy [19], which showed an increase in solubility of a water insoluble drug, namely meloxicam, with increasing PVP concentrations. The type of drugpolymer dispersion has been shown to have some promising effect on enhancement of the solubility. Comparing the three formulation types, SD's and LD's had a similar effect on solubility, increasing the solubility by approximately the same degree. The exact mechanism is unknown but it could be related to the solubilizing effect of the hydrophilic carrier, PVP in this case, and formation of eutectic mixtures [25]. Eutectic mixtures formed as a result of solid dispersions have the capability to increase the wettability of poorly water-soluble drugs and to present a microenvironment in which drug can reside in crystalline, amorphous or both forms [25]. Overall, the increase in solubility is a result of a decrease in particle size during formulation, increase in wettability and decrease in crystallinity of ART by the addition of PVP [26,27]. Previous reports have shown similar results, with PVP resulting in enhanced solubility and

Table 2 Solubility of ART from PM

\begin{tabular}{|c|c|c|c|c|c|c|}
\hline \multirow{2}{*}{ Sample } & \multicolumn{2}{|c|}{ In water } & \multicolumn{2}{|c|}{ In PBS } & \multicolumn{2}{|c|}{ In methanol } \\
\hline & Solubility (mg/mL) & $\mathrm{ER}_{\text {sol }}$ & Solubility (mg/mL) & $\mathrm{ER}_{\text {sol }}$ & Solubility (mg/mL) & $\mathrm{ER}_{\text {sol }}$ \\
\hline $\mathrm{C}$ & 0.013 & - & 0.016 & - & 16.36 & - \\
\hline $\mathrm{F} 1$ & 0.016 & 1.2 & 0.016 & 1.0 & 20.22 & 1.2 \\
\hline $\mathrm{F} 2$ & 0.018 & 1.4 & 0.017 & 1.1 & 23.71 & 1.4 \\
\hline F3 & 0.027 & 2.1 & 0.025 & 1.6 & 39.46 & 2.4 \\
\hline $\mathrm{F} 4$ & 0.031 & 2.4 & 0.037 & 2.3 & 49.12 & 3.0 \\
\hline F5 & 0.035 & 2.7 & 0.047 & 2.9 & 61.88 & 3.8 \\
\hline
\end{tabular}

Table 3 Solubility of ART from SD

\begin{tabular}{|c|c|c|c|c|c|c|}
\hline \multirow{2}{*}{ Sample } & \multicolumn{2}{|c|}{ In water } & \multicolumn{2}{|c|}{ In PBS } & \multicolumn{2}{|c|}{ In methanol } \\
\hline & Solubility (mg/mL) & $\mathrm{ER}_{\text {sol }}$ & Solubility (mg/mL) & $\mathrm{ER}_{\text {sol }}$ & Solubility (mg/mL) & $\mathrm{ER}_{\text {sol }}$ \\
\hline F6 & 0.035 & 2.7 & 0.046 & 2.8 & 67.65 & 4.0 \\
\hline F7 & 0.048 & 3.7 & 0.048 & 3.0 & 97.25 & 5.8 \\
\hline F8 & 0.059 & 4.5 & 0.052 & 3.3 & 103.70 & 6.2 \\
\hline F9 & 0.063 & 4.8 & 0.054 & 3.4 & 104.80 & 6.3 \\
\hline F10 & 0.064 & 4.9 & 0.059 & 3.7 & 139.53 & 8.4 \\
\hline
\end{tabular}

Table 4 Solubility of ART from LD

\begin{tabular}{|c|c|c|c|c|c|c|}
\hline \multirow{2}{*}{ Sample } & \multicolumn{2}{|c|}{ In water } & \multicolumn{2}{|l|}{ In PBS } & \multicolumn{2}{|c|}{ In methanol } \\
\hline & Solubility (mg/mL) & $\mathrm{ER}_{\text {sol }}$ & Solubility (mg/mL) & $\mathrm{ER}_{\text {sol }}$ & Solubility (mg/mL) & $\mathrm{ER}_{\text {sol }}$ \\
\hline F11 & 0.016 & 1.2 & 0.050 & 3.1 & 31.01 & 1.8 \\
\hline F12 & 0.021 & 1.6 & 0.057 & 3.5 & 59.75 & 3.6 \\
\hline F13 & 0.026 & 2.0 & 0.065 & 4.1 & 67.65 & 4.1 \\
\hline F14 & 0.031 & 2.4 & 0.068 & 4.2 & 109.75 & 6.6 \\
\hline F15 & 0.052 & 4.0 & 0.072 & 4.5 & 130.58 & 7.9 \\
\hline
\end{tabular}


dissolution because of decreased crystallinity of water insoluble drugs [19,28,29].

To confirm the effect of PVP on crystallinity of ART and any possible interactions, the drug-polymer dispersions were characterized by XRD and FTIR. Figure 2 displays the XRD patterns of pure ART and drug-polymer dispersions prepared by different methods. ART is a highly crystalline powder and has characteristic sharp peaks at a diffraction angle of $2 \theta$ at $7.21^{\circ}, 11.96^{\circ}, 14.54^{\circ}, 20.24^{\circ}, 21.92^{\circ}$, and $32.27^{\circ}$. The peak appearing at a diffraction angle of $2 \theta$ at $11.96^{\circ}$ is most prominent. The X-ray diffraction data for ART-PVP dispersions showed a gradual decrease in the peak intensities with increasing PVP content, which suggests either ART has lost its crystallinity and become amorphous through size reduction during formulation or the drug has been transported into the carrier during formulation. The latter aspect was more prominent in the case of $\mathrm{LD}$, where elevation of the base line was seen at the highest concentration of PVP (F15 in Figure 2). PMs showed the least effect of PVP on crystallinity of ART and the peak intensities for all PM ratios were similar to pure ART, with no identifiable changes observed (data not shown).

The interaction between the drug and carrier often leads to peculiar changes in the FTIR profile. FTIR spectra of selected formulations were compared with the standard spectra of ART and PVP-K30, as shown in Figure 3. In the functional group region, a broad band was observed at 3885 $\mathrm{cm}^{-1}$ and attributed to the presence of water because of the
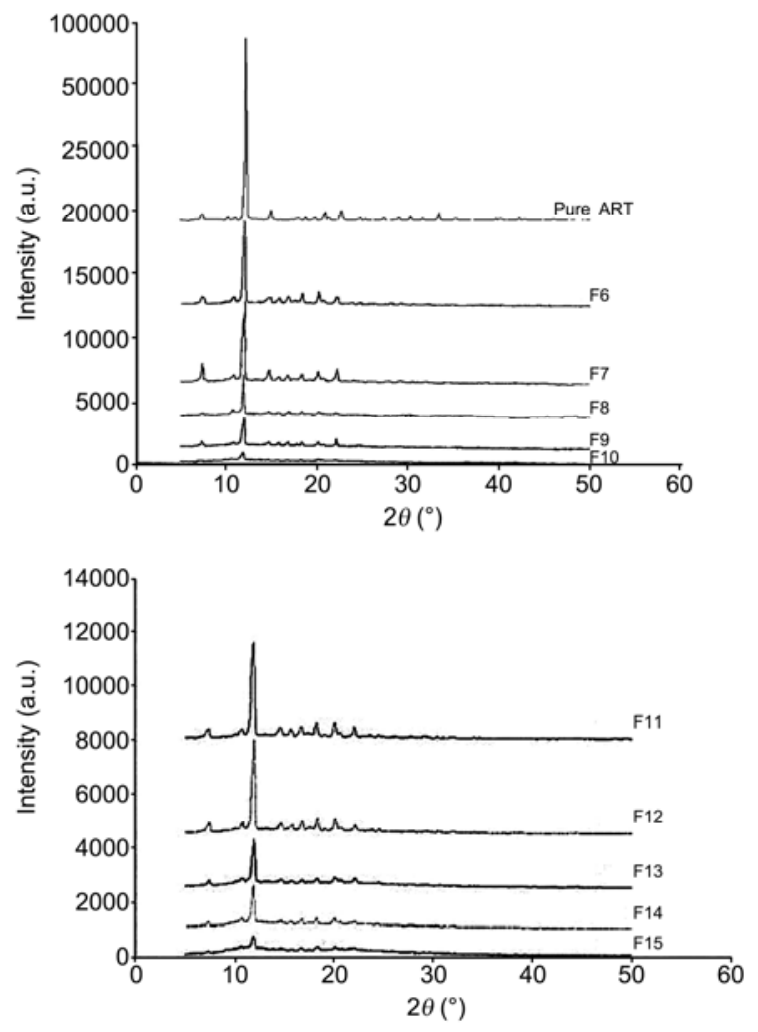

Figure 2 XRD patterns for SD and LD.

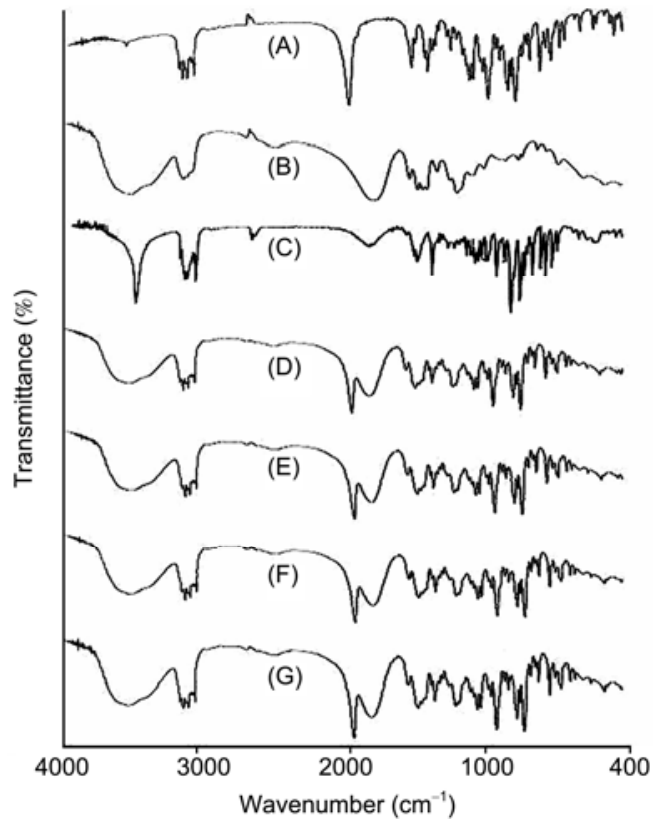

Figure 3 FTIR spectra of pure ART (A), PVP-K30 (B), F5 (C), F6 (D), F10 (E), F11 (F), F15 (G).

hygroscopic nature of PVP. A band at $876 \mathrm{~cm}^{-1}(\mathrm{O}-\mathrm{O}-\mathrm{C}$ linkage), which is characteristic of ART, was observed without shifting. All the characteristic FTIR peaks of ART were visible without shifting in all formulation systems, suggesting an absence of significant interactions between PVP and ART.

In vitro diffusion across a silicone membrane was studied for all formulations. The steady-state flux was determined from the slope of the linear portion of the cumulative amount of drug permeation versus time plot, as shown in Figure 4. Permeability and diffusion coefficients were calculated by applying Fick's laws of diffusion. The permeation profile and flux enhancement ratio (ER) are summarized in Table 5. The cumulative amount of drug permeated through the silicone membrane as a function of polymer ratio revealed that PVP increased the rate of permeation markedly for each formulation system compared to pure ART. In the case of SD, F10 showed a maximum amount of drug permeation, while a gradual increase in permeation of ART was observed for LD with increasing PVP concentration. Although there was no significant difference in drug permeation for F10 and F15, ART permeation from LD formulations with a low PVP fraction (F1, F2, F3 and F4) differed significantly from similar ratios in SD formulations. The lyophilization process involves simultaneous primary and secondary drying, which results in a porous and fluffy dry mixture [30]. This increases the surface area and surface free energy and therefore drug molecules penetrate into membrane faster than usual. This is the reason that the initial permeation of ART was higher in the LD system than SD. Physical mixtures showed the lowest permeation enhancement because of the crystalline nature of ART, which 

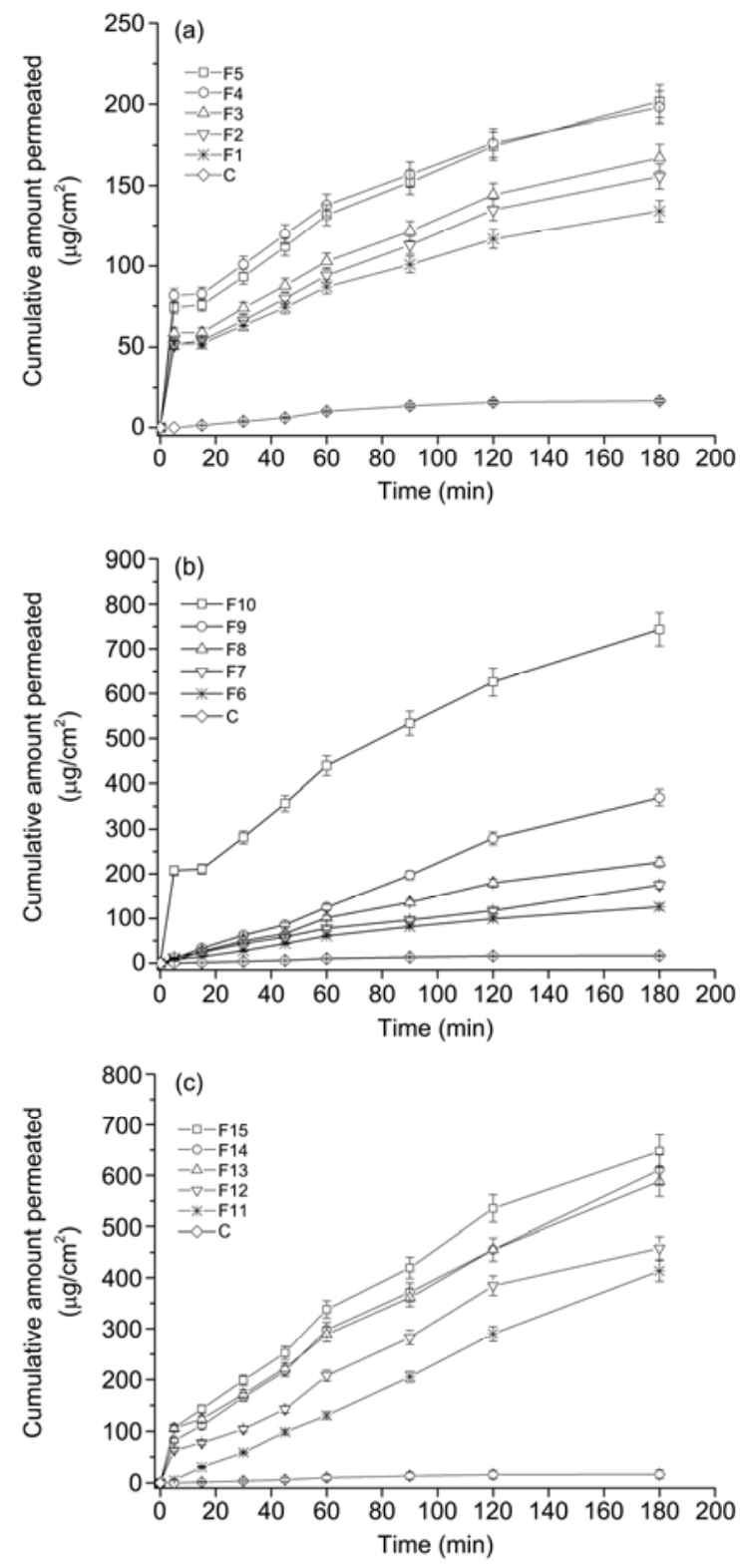

Figure 4 Cumulative drug permeated from PMs (a); SDs (b); LDs (c).

was confirmed by XRD. The flux values increased with increasing PVP concentration, suggesting that PVP enhanced the solubility of ART and solvent uptake in the silicone membrane.

Figure 5(a) and (b) displays the relationship between the flux enhancement ratio and the solubility enhancement ratio, which suggests that an increase in solubility with varying concentration of PVP also increased the flux. It has been reported that the concentration of enhancer in a system under investigation can influence the promotion of transdermal drug delivery [31,32]. Thus, the amount of permeation enhancer present in the membrane is an important factor in the increase in permeation rate [33]. The saturated solutions of PM's, SD's and LD's were provided with a sufficient excess of solute (ART) to maintain a constant donor concen-

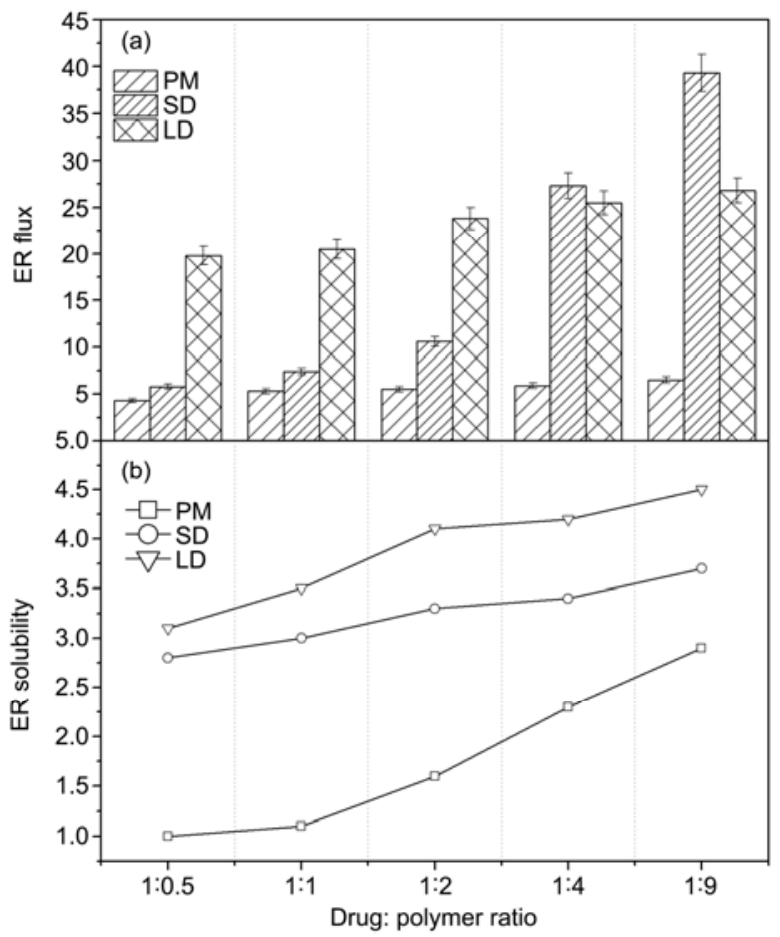

Figure 5 Comparison of ER flux (a) and ER solubility (b).

tration throughout the course of experiment for all formulation systems. When equilibrium conditions are maintained, the flux will be maximized when the outer layer of the membrane is saturated with the solute. According to Dias and co-workers [34], ideally all saturated solutions of the same permeant in any solvent system should produce an equal flux through a membrane, independent of solute concentration. Examination of the values in Table 5 reveals that the flux across the silicone membrane obtained here was not constant but increased with increasing PVP-K30 concentration. This may be due to some or all of the situations examined here being non-ideal, i.e. there may have some interaction between the vehicle and membrane that increased the diffusion coefficient of the solute within the membrane [35].

The permeability coefficients as a function of PVP concentration revealed its dependence on the amount of polymer in the formulation. The increased permeability coefficient can be the result of two factors, namely, physicochemical characteristics and reduction in drug crystallinity. First, ART is lipophilic in nature, which creates a more favorable environment for the interaction with the silicone membrane, which is also lipophilic [36]. The increased solubility of ART in the internal lipophilic environment of the silicone membrane resulted in an enhanced permeability coefficient. To some extent, the crystallinity of drugs affects drug solubility and therefore, attenuation of drug crystallization by polymers has been used for many pharmaceutical applications over many years [37]. PVP has a tendency to hamper the re-crystallization of drugs, thus amorphous forms 
Table 5 Permeation profile of ART from different formulations ${ }^{\text {a) }}$

\begin{tabular}{|c|c|c|c|c|}
\hline Formulation & $\begin{array}{c}\text { Flux }(\mathrm{J}) \\
\left(\mu \mathrm{g} /\left(\mathrm{cm}^{2} \mathrm{~min}\right)\right) \\
\end{array}$ & $\begin{array}{l}\text { Diffusion coefficient } \\
\left(10^{-4} \times D\right)\left(\mathrm{cm}^{2} / \mathrm{min}\right)\end{array}$ & $\begin{array}{c}\text { Permeability coefficient } \\
\left(10^{-4} \times K_{\mathrm{p}}\right)(\mathrm{cm} / \mathrm{min})\end{array}$ & $\begin{array}{c}\text { Enhancement ratio } \\
\operatorname{ER}(\mathrm{J}) \\
\end{array}$ \\
\hline $\mathrm{C}$ & $0.12 \pm 0.01$ & $1.81 \pm 0.01$ & $65.6 \pm 4.15$ & - \\
\hline $\mathrm{F} 1$ & $0.51 \pm 0.03$ & $1.33 \pm 0.29$ & $2.69 \pm 0.13$ & 4.3 \\
\hline $\mathrm{F} 2$ & $0.64 \pm 0.10$ & $1.07 \pm 0.12$ & $3.34 \pm 0.50$ & 5.3 \\
\hline F3 & $0.66 \pm 0.03$ & $1.08 \pm 0.20$ & $3.41 \pm 0.16$ & 5.5 \\
\hline F4 & $0.71 \pm 0.42$ & $1.26 \pm 0.41$ & $4.24 \pm 2.12$ & 5.9 \\
\hline F5 & $0.78 \pm 0.30$ & $1.06 \pm 0.89$ & $4.98 \pm 1.51$ & 6.5 \\
\hline F6 & $0.70 \pm 0.01$ & $1.83 \pm 0.60$ & $3.51 \pm 0.02$ & 5.8 \\
\hline F7 & $0.89 \pm 0.01$ & $2.11 \pm 0.10$ & $4.50 \pm 0.48$ & 7.4 \\
\hline F8 & $1.28 \pm 0.24$ & $1.08 \pm 0.56$ & $6.43 \pm 1.20$ & 10.6 \\
\hline F9 & $3.28 \pm 0.09$ & $0.12 \pm 0.38$ & $16.39 \pm 0.49$ & 27.3 \\
\hline F11 & $2.37 \pm 0.05$ & $0.98 \pm 0.21$ & $11.86 \pm 0.27$ & 19.8 \\
\hline F12 & $2.46 \pm 0.05$ & $2.70 \pm 0.16$ & $12.28 \pm 0.29$ & 20.5 \\
\hline F13 & $2.85 \pm 0.01$ & $4.22 \pm 0.57$ & $14.27 \pm 0.06$ & 23.8 \\
\hline F14 & $3.06 \pm 0.49$ & $3.40 \pm 0.38$ & $15.33 \pm 2.49$ & 25.5 \\
\hline F15 & $3.21 \pm 0.07$ & $4.46 \pm 0.78$ & $16.07 \pm 0.34$ & 26.8 \\
\hline
\end{tabular}

a) Results are presented as mean $\pm \operatorname{SD}(n=3)$.

of drugs show an enhanced permeability [38]. The increase in permeability coefficient with an increasing PVP content reflects a reduction in crystallinity that, in turn, improves the permeation of ART across the silicone membrane. XRD patterns confirmed a prominent decrease in crystallinity with increasing polymer content in the cases of SD and LD.

In conclusion, the effect of PVP on ART solubility and in vitro diffusion was evaluated by making PMs, SDs and LDs at varying concentrations of PVP-K30. Results showed that there is a polymer-dependent increase in solubility and permeation of drug across the model membrane. Higher concentrations of PVP result in higher solubility and permeation. This enhancement was attributed to many factors, including a decrease in crystallinity of ART, an increase in solute wettability and the formation of eutectic mixtures of drug in the SD and LD systems. Solid state properties studied by XRD confirmed the loss of crystallinity in the cases of SD and LD formulation systems, while no interaction between ART and PVP was observed using FTIR. The increase in pharmaceutical properties of ART was in the order of $\mathrm{LD}>\mathrm{SD}>\mathrm{PM}$. Future work will focus on making an optimized formulation for transdermal delivery.

The authors are thankful to Bahauddin Zakariya University and the Higher Education Comission, Pakistan, for providing funding (628) to conduct this research.

1 Svensson U S H, Sandström R, Carlborg Ö, et al. High in situ rat intestinal permeability of artemisinin unaffected by multiple dosing and with no evidence of $P$-glycoprotein involvement. Drug Metab Dispos, 1999, 27: 227-232
2 Thaithong S, Beale G H. Susceptibility of Thai isolates of Plasmodium falciparum to artemisinine [qinghaosu] and artemether. Bull World Health Organ, 1985, 63: 617-619

3 Klayman D L. Qinghaosu [artemisinin]: An antimalarial drug from China. Science, 1985, 228: 1049-1055

4 Titulaer H, Zuidema J, Kager P, et al. The pharmacokinetics of artemisinin after oral, intramuscular and rectal administration to volunteers. J Pharma Pharmacol, 1990, 42: 810-813

5 Wong J, Yuen K. Improved oral bioavailability of artemisinin through inclusion complexation with (beta)-and (gamma)-cyclodextrins. Int J Pharm, 2001, 227: 177-185

6 Sahoo N G, Kakran M, Li L, et al. Dissolution enhancement of a poorly water-soluble antimalarial drug by means of a modified multifluid nozzle pilot spray drier. Mat Sci Eng C, 2011, 31: 391-399

7 Stoughton R B. Percutaneous absorption. South Med J, 1962, 55: 1134-1138

8 Kim J H, Choi H K. Effect of additives on the crystallization and the permeation of ketoprofen from adhesive matrix. Int J Pharm, 2002, 236: $81-85$

9 Barry B W. Skin transport. In: Dermatological Formulations Percutaneous Absorption. New York: Marcel Dekker, 1983. 95-126

10 Iwasa A, Irimoto K, Kasai S, et al. Effect of nonionic surfactants on percutaneous absorption of diclofenac sodium. Yakuzaigaku, 1991, 51: $16-21$

11 Javadzadeh Y, Shokri J, Hallaj-Nezhadi S, et al. Enhancement of percutaneous absorption of Finasteride by cosolvents, cosurfactant and surfactants. Pharm Dev Technol, 2009: 1-7

12 Pathan I B, Setty C M. Chemical penetration enhancers for transdermal drug delivery systems. Trop J Pharm Res, 2009, 8: 173-179

13 Karavas E, Ktistis G, Xenakis A, et al. Effect of hydrogen bonding interactions on the release mechanism of felodipine from nanodispersions with polyvinylpyrrolidone. Eur J Pharm Biopharm, 2006, 63: 103-114

14 Ruan L P, Yu B Y, Fu G M, et al. Improving the solubility of ampelopsin by solid dispersions and inclusion complexes. J Pharm Biomed Anal, 2005, 38: 457-464

15 Tantishaiyakul V, Kaewnopparat N, Ingkatawornwong S. Properties of solid dispersions of piroxicam in polyvinylpyrrolidone K-30. Int J 
Pharm, 1996, 143: 59-66

16 Van Nijlen T, Brennan K, Van den Mooter G, et al. Improvement of the dissolution rate of artemisinin by means of supercritical fluid technology and solid dispersions. Int J Pharm, 2003, 254: 173-181

17 Akbuga J, Gürsoy A, Kendi E. The preparation and stability of fast release furosemide-PVP solid dispersion. Drug Dev Ind Pharm, 1988, 14: 1439-1464

18 Margarit M V, Marín M T, Contreras M D. Solubility of solid dispersions of pizotifen malate and povidone. Drug Dev Ind Pharm, 2001, 27: 517-522

19 El-Badry M, Fathy M. Enhancement of the dissolution and permeation rates of meloxicam by formation of its freeze-dried solid dispersions in polyvinylpyrrolidone K-30. Drug Dev Ind Pharm, 2006, 32: $141-150$

20 Zhao S S, Zeng M Y. Spektrometrische Hochdruck-FlüssigkeitsChromatographische [HPLC] Untersuchungen zur Analytik von Qinghaosu. Planta Med, 1985, 51: 233-237

21 Ansari M T, Iqbal I, Sunderland V B. Dihydroartemisinin-cyclodextrin complexation: Solubility and stability. Arch Pharmacal Res, 2009, 32: $155-165$

22 Franz T J. Percutaneous absorption: On the relevance of in vitro data. J Invest Dermatol, 1975, 64: 190-195

23 Avery M A, Bonk J D, Chong W K M, et al. Structure-activity relationships of the antimalarial agent artemisinin. 2. Effect of heteroatom substitution at O-11: Synthesis and bioassay of $\mathrm{N}$-alkyl-11aza-9-desmethylartemisinins. J Med Chem, 1995, 38: 5038-5044

24 Sammour O A, Hammad M A, Megrab N A, et al. Formulation and optimization of mouth dissolve tablets containing rofecoxib solid dispersion. AAPS PharmSciTech, 2006, 7: 167-175

25 Chiou W L, Riegelman S. Pharmaceutical applications of solid dispersions. J Pharm Sci, 1971, 60: 1281-1302

26 Abdelkader H, Abdallah O Y, Salem H S. Comparison of the effect of tromethamine and polyvinylpyrrolidone on dissolution properties and analgesic effect of nimesulide. AAPS PharmSciTech, 2007, 8: $110-117$
27 Shah J, Vasanti S, Anroop B, et al. Enhancement of dissolution rate of valdecoxib by solid dispersions technique with PVP K 30 \& amp; PEG 4000: Preparation and in vitro evaluation. J Incl Phen Macro, 2009, 63: 69-75

28 Liu C, Desai K G H. Enhancement of dissolution rate of valdecoxib using solid dispersions with polyethylene glycol 4000. Drug Dev Ind Pharm, 2005, 31: 1-10

29 Modi A, Tayade P. Enhancement of dissolution profile by solid dispersion [kneading] technique. AAPS PharmSciTech, 2006, 7: 87-92

30 Betageri G V, Makarla K R. Enhancemnet of dissolution of glyburide by solid dispersion and lyophilisation techniques. Int J Pharm, 1995, 126: $155-160$

31 Barry B W. Properties that influence. In: Dermatological Formulations Percutaneous Absorption. New York: Marcel Decker, 1983. 127-233

32 Chow D. Concentration dependent enhancement of 1-dodecylazacycloheptan-2-one on the percutaneous penetration kinetics of triamcinolone acetonide. J Pharm Sci, 1984, 73: 1794-1799

33 Huang Y B, Wu P C, Ko H M, et al. Cardamom oil as a skin permeation enhancer for indomethacin, piroxicam and diclofenac. Int $\mathbf{J}$ Pharm, 1995, 126: 111-117

34 Dias M, Hadgraft J, Lane M E. Influence of membrane-solvent-solute interactions on solute permeation in model membranes. Int J Pharm, 2007, 336: 108-114

35 Watkinson A C, Joubin H, Green D M, et al. The influence of vehicle on permeation from saturated solutions. Int J Pharm, 1995, 121: 27-36

36 Raghavan S, Kiepfer B, Davis A, et al. Membrane transport of hydrocortisone acetate from supersaturated solutions; the role of polymers. Int J Pharm, 2001, 221: 95-105

37 Kim J H, Lee C H, Choi H K. Transdermal delivery of physostigmine: Effects of enhancers and pressure-sensitive adhesives. Drug Dev Ind Pharm, 2002, 28: 833-839

38 Zhang J, Liu Z, Du H, et al. A novel hydrophilic adhesive matrix with self-enhancement for drug percutaneous permeation through rat skin. Pharm Res, 2009, 26: 1398-1406

Open Access This article is distributed under the terms of the Creative Commons Attribution License which permits any use, distribution, and reproduction in any medium, provided the original author(s) and source are credited. 